\title{
EDITORIAL
}

\section{SOBRE LOS META-ANÁLISIS Y EL QUOROM}

\author{
Fernando Rodríguez Artalejo (1) y Pilar Guallar Castillón (2)
}

(1) Departamento de Medicina Preventiva y Salud Pública. Universidad Autónoma. Madrid

(2) Ccntro Universitario de Salud Pública. Universidad Autónoma de Madrid y Consejería de Sanidad de Madrid.

Las revisiones sistemáticas (RS) de la literatura científica y los meta-análisis, un tipo de RS en la que se usan técnicas estadísticas, son piezas clave en el ejercicio de la medicina y la salud pública basadas en la evidencia. Sin embargo, la calidad de las RS de ensayos clínicos es claramente mejora$b^{1} e^{1,2}$, por lo que un grupo de investigadores y usuarios de meta-análisis han publicado recientemente en Lancet unas recomendaciones (identificadas por el acrónimo $Q U O$ $R O M$ ) para contribuir a resolver dicho problema $^{3}$. Si esta iniciativa tiene éxito y es asumida por las revistas científicas más importantes, tal como ocurrió con su antecedente para los ensayos clínicos (el conocido $(O N S O R T)^{4}$, dichas recomendaciones pueden convertirse en norma para publicar nuestras RS. Por todo ello, es una buena ocasión para reflexionar sobre la metodología de elaboración de las recomendaciones QUOROM y sobre la contribución de las RS a la toma de decisiones clínicas y de salud pública. A continuación aparecen algunos comentarios.

1. Llama la atención que se hayan utilizado métodos bastante cualitativos y subjetivos (como una modificación del Delphi)

Correspondencia:

Dr. Fernando Rodríguez Artalejo

Facultad de Medicina

Departamento de Medicina Preventiva y Salud Pública.

Universidad Autónoma de Madrid.

Avda. Arzobispo Morcillo, s/n

28029 Madrid

Correo electrónico: remando.artalejo@uam.es para elaborar las recomendaciones $Q U O$ $R O M$, que se refieren a los meta-análisis, las técnicas más cuantitativas y objetivas para la revisión del conocimiento científico. Además, en el resumen del trabajo, los autores destacan que se trata de su forma 'preferida' para presentar los componentes de un meta-análisis. Ello traduce dos cosas. Primero, que no hay muchas evidencias (y las disponibles son mayoritariamente indirectas) sobre las intervenciones más eficaces en este campo. Segundo, que donde no hay evidencias, pucde que lo razonable no siempre sea abstenerse de hacer una recomendación. Si ésta no parece ser dañina, y está basada en el sentido común (como son muchas de las del $Q U O R O M$ ), puede llegar a funcionar bien.

2. También es llamativo que se haya tardado tres años en publicar la declaración QUOROM. El trabajo se realizó básicamente en 1996 y en la publicación no se informa suficientemente de las razones para la espera. Este tiempo podría haberse utilizado para lograr la adhesión de la Colaboración Cochrane (de hecho las recomendaciones fueron testadas en uno de los centros Cochrane de Canadá) o para integrarse en las normas de publicación de algunas revistas de prestigio (empezando por el Lancet, donde se publica el QUOROM).

3. La declaración $Q U O R O M$ parece hacer excesivo énfasis en la síntesis estadística de los resultados de los ensayos clínicos. Es cierto que es el componente estadístico el 
que define a los meta-análisis dentro de las RS. Sin embargo, a menudo lo más informativo es poner de manifiesto discrepancias entre los estudios «meta-analizados», identificar sus causas, y sugerir una pequeña agenda de investigación en la que se identifiquen preguntas todavía no respondidas y se mejore la calidad de los futuros trabajos.

4. Una RS no es sinónimo de calidad. Las hay buenas, y las hay muy mejorables. Atendemos a numerosas presentaciones en las que se invoca el término RS o meta-análisis para reivindicar la calidad de los hallazgos que se presentan, sin que el ponente se detenga a valorar críticamente dichas RS. Como toda investigación retrospectiva, las RS tienen limitaciones, principalmente en la calidad de la información disponible. Además la reproducibilidad de algunas RS no es buena $^{5}$, y a veces sus resultados no coinciden con los de los grandes ensayos clínicos ${ }^{6}$. Lo que es peor, a menudo no está claro qué es más cierto, el resultado de la RS de pequeños ensayos clínicos (que suele tener alguna validez externa, porque se han realizado con poblaciones y métodos variados) o el de un gran ensayo clínico (posiblemente con mayor validez interna).

5. Por otro lado, la calidad del informe no es igual a la calidad (validez interna) del meta-análisis. Esto depende más de la valía «intrínseca» del científico que de la medida en que se explicitan sus métodos (a lo que se dedica principalmente la declaración QUOROM). Para lo primero, hay que conocer tanto las técnicas de la revisión sistemática, como el tema de estudio, y tener experiencia investigadora. Ello no se consigue simplemente con guías o recomendaciones, aunque algo puedan ayudar. Utilizando un símil culinario, hacer una buena paella es bastante más (difícil) que disponer de una receta y echar sobre el fuego todos sus componentes en el orden y durante el tiempo adecuado.

6. La declaración $Q U O R O M$ se limita a los ensayos clínicos. Sin embargo, estos es- tudios a menudo no son los más útiles para informar las decisiones de salud pública ${ }^{7}$. A pesar de los esfuerzos y numerosos métodos disponibles para valorar la calidad de los informes de estudios observacionales, y sus RS, todavía no hay un método consensuado para ello. Probablemente se debe a la dificultad de estandarizar la realización de dichos trabajos y sus revisiones (por no hablar de la medida de su validez interna).

7. En el campo de la epidemiología clínica abundan las reglas y recomendaciones, desde cómo leer o usar muchos tipos de artículos $^{8}$, hasta qué información proporcionar en la publicación de un ensayo clínico ${ }^{4}$ o más recientemente una $\mathrm{RS}^{3}$. No deben ser una traba para dejar volar la imaginación (algunas de las mejores revistas científicas siguen teniendo un formato libre) o para el escrutinio con inteligencia, más que con método, de cada pieza de la literatura.

8. Por último, la «interpretación» del resumen de la declaración QUOROM es una invitación al desarrollo de la epidemiología de las RS y los meta-análisis. El estudio sistemático de las RS (es una buena redundancia) nos hará conocer mejor las características de las mejores revisiones, de forma que es presumible que las actualizaciones del QUOROM estén más basadas en evidencias. Lo ahora disponible parece sensato, aunque sea sobre todo una guía para la publicación de RS basada principalmente en una revisión narrativa (por ello con una proporción variable de evidencias y opiniones). Como vemos, las revisiones narrativas siguen siendo útiles para abordar temas amplios en los que el sesgo (entendido no como error epidemiológico sino como inclinación u orientación) de los expertos puede resultar interesante, e incluso útil.

\section{BIBLIOGRAFÍA}

1. Sacks HS, Berrier J, Reitman D, Ancona-Berk VA, Chalmers TC. Meta-analyses of randomized controlled trials. N Engl J Med 1987; 316 : 450-455. 
2. Sacks HS, Reitman D, Pagano D, Kupelnick B. Meta-analysis: an update. Mt Sinai J Mcd 1996; 63: 216-224.

3. Moher D, Cook DJ, Eastwood S, et al for the QUOROM Group. Improving the quality of reports of meta-analyses of randomised controlled trials: the QUOROM statement. Lancet 1999; 354: 1896-1900.

4. Begg C, Cho M, Eastwood S, et al. Improving the quality of reporting of randomized controlled trials. The CONSORT statcment. JAMA 1996; 276: 637-639.
5. Jadad AR, Cook DJ, Browman G. A guide to interpreting discordant systematic reviews. Can Med Assoc J 1997; 156: 1411-1416.

6. LeLorier J, Gregroire G, Benhaddad A, Lapierre J, Derderian F. Discrepancies between metaanalyses and subsequent large randomized, controlled trials. N Engl J Med 1997; 337: 536-542.

7. Rodríguez Artalejo F. La salud pública basada en la evidencia. Gac Sanit 1997; 11: 201-203.

8. La medicina basada en la evidencia. Guías del usuario de la literatura médica. JAMA edición española. Barcelona: PPI, 1997. 